University of Nebraska - Lincoln

DigitalCommons@University of Nebraska - Lincoln

6-2013

\title{
Qigong As A Novel Intervention For Service Members With Mild Traumatic Brain Injury
}

Terri L. Yost

Tripler Army Medical Center, terri.yost@us.army.mil

Ann Gill Taylor

University of Virginia

Follow this and additional works at: https://digitalcommons.unl.edu/usarmyresearch

Yost, Terri L. and Gill Taylor, Ann, "Qigong As A Novel Intervention For Service Members With Mild Traumatic Brain Injury" (2013). US Army Research. 236.

https://digitalcommons.unl.edu/usarmyresearch/236

This Article is brought to you for free and open access by the U.S. Department of Defense at DigitalCommons@University of Nebraska - Lincoln. It has been accepted for inclusion in US Army Research by an authorized administrator of DigitalCommons@University of Nebraska - Lincoln. 


\section{Qigong as a Novel Intervention for Service Members With Mild TRAUMATIC BRAIN INJURY

\author{
Terri L. Yost, PhD, FNP-BC, ${ }^{1 \#}$ and Ann Gill Taylor, EdD, MS, RN, FAAN ${ }^{2}$
}

\begin{abstract}
Purpose: To describe the experience of internal qigong practice in service members diagnosed with mild traumatic brain injury (mTBI).
\end{abstract}

Theoretical Framework: The study used qualitative descriptive phenomenological methods originally described by Husserl and later refined by Giorgi.

Methodology: Participants were interviewed about their experiences while learning qigong to determine their level of interest, benefits, and/or adverse effects; ease of learning/performing the routine; and any barriers to practice.

Sample: Six service members with mTBI receiving outpatient neurorehabilitation at the Defense and Veterans Brain Injury Center-Charlottesville Rehabilitation Center.

Intervention: Participants learned Reflective Exercise Qigong, a form of qigong developed specifically to require less complex movement and balance than most forms of qigong, making it ideal for those with potential coordination and balance issues.
Data Collection: Semistructured interviews took place after four weeks of formal qigong instruction, then again after the subjects completed eight weeks. Interview data were analyzed with phenomenological methods described by Giorgi.

Results: Four themes emerged from the interview data: "the physical experience of qigong," "regaining control," "no pain, a lot of gain," and "barriers to qigong practice." Participants offered examples of how qigong enabled them to control refractory symptoms after mTBI while decreasing reliance on pharmacotherapy. All agreed that qigong was uniquely conducive to the disciplined mindset of military service members and that the simplicity of Reflective Exercise qigong, compared with similar modalities such as tai chi and yoga, was well suited to individuals with decreased balance, cognition, and memory related to mTBI.

Key words: Mild traumatic brain injury, mTBI, qigong, reflective exercise, phenomenology, military

(Explore 2013; 9:142-149. Published by Elsevier Inc.)

\section{INTRODUCTION}

Traumatic brain injury (TBI) is a topic for which its relevance cannot be overstated. Journals, newspapers, magazines, and Web sites reveal new insights and information on the significance of TBI and concussions on a near-daily basis as it pertains to the populations of youth and professional athletes as well as military service members. Studies report that the prevalence of TBI in service members returning from deployment in either Iraq or Afghanistan is as great as $22.8 \%{ }^{1}$-a rate for TBI that has caused a call to arms in the military community.

It is estimated that $70 \%$ of all recent combat-related injuries are the result of a blast ${ }^{2}$ and that approximately $60 \%$ of these blast exposures may result in a brain injury. ${ }^{3}$ The vast majority of battle-related TBIs are mild in nature and may result in symptoms such as headache, depression, memory loss, sleep disturbances, personality changes, anger, communication disorders, cognitive deficits, vestibular disorders, and tinnitus. ${ }^{4}$

1 Center for Nursing Science and Clinical Inquiry, Tripler Army Medical Center, Honolulu, HI

2 Center for the Study of Complementary and Alternative Therapies, University of Virginia, Charlottesville, VA

\# Corresponding Author. Address:

Center for Nursing Science and Clinical Inquiry, Tripler Army Medical Center, 1 Jarrett White Road, Honolulu, HI 96859

e-mail: terri.yost@us.army.mil
The invisibility of these symptoms adds to the burden of thousands of service members and their loved ones as they attempt to adapt to life after a mild traumatic brain injury (mTBI). ${ }^{5}$

Military healthcare providers find the complex nature of mTBI-related symptoms challenging to manage. Thus, to help promote consistent and uniform treatment for mTBI among all military and veteran treatment facilities, the Department of Defense and the Department of Veterans Affairs collaborated to develop a clinical practice guideline for the management of mTBI. ${ }^{4}$ This evidence-based guideline features practice algorithms and treatment recommendations with accompanying evidence statements. However, the strongest evidence-based recommendations from the guideline focus on the general approach of normalizing symptoms through the use of education while managing physical symptoms through a piecemeal approach. Consequently, care is fragmented among primary care providers, mental health services, and medical specialists, ${ }^{6}$ resulting in other problems such as polypharmacy. ${ }^{7}$ Polypharmacy often leads to a decreased compliance with established treatment regimens, further reducing the effectiveness of symptom management in some service members. ${ }^{8}$ To reduce the consequences of fragmented medical care, some healthcare providers are calling for a new framework for healing that incorporates the relationship between the mind and the body. ${ }^{9}$ The goal of such a framework is to foster interventions for healing that are safe, have few side effects, and address multiple symptoms effi- 


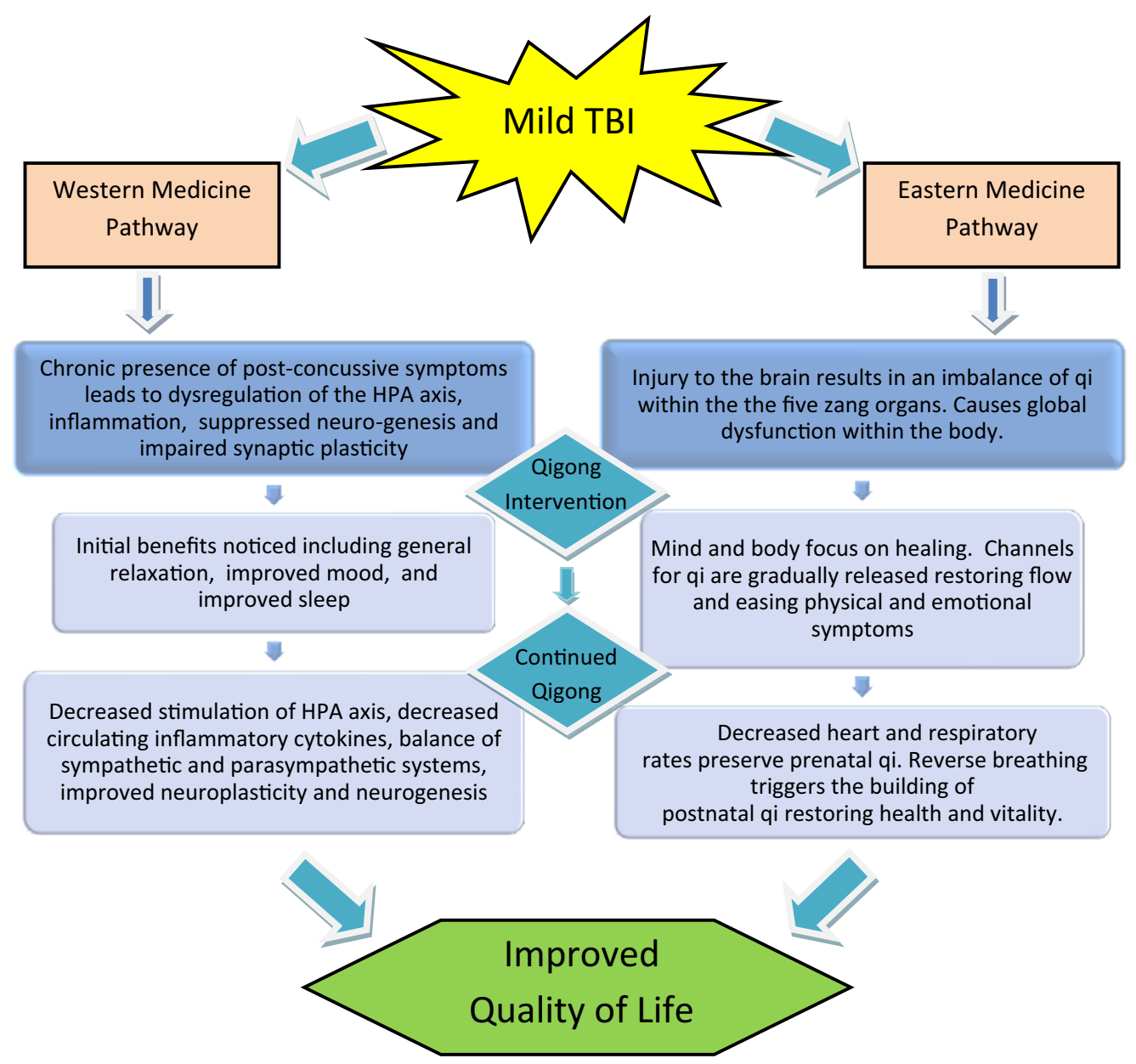

Figure 1. Proposed Western medicine and Eastern medicine pathways of qigong.

ciently. One such mind-body modality with this potential is qigong.

Qigong is an ancient Chinese healing art based in eastern philosophy that has been practiced for thousands of years. Although hundreds of different styles are now practiced worldwide, all consist of three basic components: controlled breathing, meditation, and smooth, continuous, purposeful movement. Qigong has been shown to reduce sympathetic activation and enhance cardiovagal tone both acutely and long-term in clinical ${ }^{10,11}$ as well as nonclinical populations. ${ }^{12}$ A growing body of research suggests that qigong, like other forms of meditation practice, may promote beneficial changes in the central nervous system, including favorable changes in dopaminergic and other neurochemical systems ${ }^{13,14}$ as well as increases in blood flow, oxygen delivery, and glucose use in specific regions of the brain associated with mood elevation, memory, and attentional processing, including the hippocampus, prefrontal cortex, and anterior cingulate gyrus. ${ }^{14}$ In addition, recent research suggests that qigong training programs can enhance immune response ${ }^{15,16}$ while reducing blood pressure, ${ }^{11}$ insulin resistance, ${ }^{17}$ oxidative stress, ${ }^{18}$ and other related indexes of cerebrovascular disease risk. ${ }^{11,17}$ Although research on TBI survivors remains limited, findings from observational studies sup- port the implementation of qigong programs in this population and suggest that qigong practice may reduce stress, anxiety, Posttraumatic stress disorder (PTSD) and depression, and improve health outcomes in adults with TBI-related symptoms. A theoretical framework illustrating the proposed mechanisms by which qigong exerts its effects on the body is shown in Figure 1.

PTSD is a common comorbidity in those who have experienced mTBI and is frequently associated with protracted postconcussive symptoms in military service members. ${ }^{5,19-21}$ Comorbid PTSD and head trauma are also common in survivors of torture. Researchers who have worked closely with refugees and survivors of torture have found general benefits and symptom reduction while engaging these survivors in the practice of qigong and tai chi. ${ }^{9}$ Also, torture victims with PTSD who practice these ancient arts have reported exhibiting fewer flashbacks and anxiety during their practice sessions than when practicing meditation alone, presumably because the meditative practices within qigong and tai chi are incorporated into active body movements. ${ }^{9}$ Given these findings, military service members with similar symptoms of mTBI and PTSD may also benefit from these mind-body therapies. In addition, ties between qigong and its martial arts forms, tai chi and kung fu, may hold a certain appeal for military service members. The purpose of 
this pilot study was to describe the experience of internal qigong practice in service members diagnosed with mTBI.

\section{METHODS}

Given the pilot nature of the study and the desire to explore the experience of qigong practice among a unique population, a qualitative methodology was chosen for its ability to obtain rich narrative data while not limiting the responses of participants through pre-established questionnaires. It was also believed that given the potential cognitive limitations of those with mTBI, qualitative data in the form of interactive interviews-the relative length and intensity of which were driven by the abilities and tolerance of the participant-would reduce study-related burden. Thus, the methodological design selected was descriptive phenomenology, originally described by Husserl ${ }^{22}$ and later refined by Giorgi. ${ }^{23}$ The aim of this approach is to describe a lived condition or experience in an effort to gain a better understanding of its essence and what it means to those experiencing it. Through this methodology, thoughtful discussions regarding the experience of qigong practice could inform possible future qigong research in military service members as well as provide valuable insight into improving the implementation of future studies from the participants' perspective.

Compared with other qualitative methods, phenomenological studies tend to use smaller sample sizes, typically 5 to 10 participants, to maintain the personal elements of the livedexperience. $^{24-26}$ Several additional factors were also considered in determining an appropriate sample size for this study. Because of the pilot nature of the study, data saturation was not the central end point. Also, the purpose and aims of the study had a very narrow focus. Because interviews were focused on the experiences of learning and practicing qigong, there was a greater likelihood that the data obtained during the interviews would be similarly focused. The study design also affected the potential sample size in that each participant was interviewed twice over the course of the intervention, thus producing more usable data for analysis. Taking these factors into consideration as well as sample size recommendations in the phenomenology literature, we set a goal of 6 to 10 participants.

Approvals to conduct the research were granted by the University of Virginia Institutional Review Board for Health Sciences Research, the Uniformed Services University of the Health Sciences Institutional Review Board, and the Headquarters of the Defense and Veterans Brain Injury Center (DVBIC) and conducted with the ethical standards set forth in the Helsinki Declaration of 1975.

Upon acceptance into the study, each participant completed a baseline questionnaire and interview and then began the formal qigong instruction. After four weeks of instruction, participants were interviewed with the use of semistructured questions to determine their initial impressions of the qigong, the ease of learning this ancient art, and any effects they perceived the qigong to have had on their recovery process. Length of the interviews ranged from 30 to 60 minutes. Audio files from the interviews were transcribed verbatim into Microsoft Word documents. This same interview process was repeated after completion of the full 8-week qigong intervention.

\section{Participants}

Participants were recruited from the Charlottesville Rehabilitation Program, a core program of the DVBIC. The DVBIC-Charlottesville is one of only two civilian facilities dedicated to the treatment of brain injury that are part of the DVBIC's large military network of care providers. DVBIC-Charlottesville's community-integrated brain injury rehabilitation program offers comprehensive evaluation, outpatient therapy, vocational training, and innovative community re-entry services for military members (and civilians to a lesser extent) with acquired or traumatic brain injuries. Flyers describing the nature of the research study were placed in the neurorehabilitation facility, and the principal investigator was invited periodically by the medical staff to attend group question and answer sessions related to the study protocol. The purposive, convenience sampling methodology used in this recruiting procedure produced the six volunteers who would participate in this qigong study.

Participants who volunteered met the following inclusion criteria: (1) military service member who had suffered mTBI during active military service, (2) receiving outpatient neurorehabilitation, (3) older than 18 years of age, (4) a history of at least one brain injury, (5) a diagnosis (by the staff of DVBIC-Charlottesville) consistent with $\mathrm{mTBI},(\mathrm{g})$ ability to speak and understand English, (7) willingness to talk about living with a brain injury, and (8) ability to provide informed consent. Potential participants were excluded if the severity of their brain injuries were categorized as moderate or severe.

\section{Intervention}

The qigong form used in this study was Reflective Exercise (RE) which was developed and taught to the participants by qigong master John Alton. In RE qigong, outcomes for the participants are a result of their own internal energy (internal qigong) as opposed to the external energy of a qigong master (external qigong). Alton developed this style specifically to require less complex movement and balance than required by most forms of qigong, making it ideal for those with potential balance and coordination issues such as individuals with mTBI. ${ }^{27}$

RE qigong comprises several core elements, including reverse abdominal breathing, the six subtle movements, and reflective meditation (both standing and seated). Full details regarding RE qigong practice are discussed in Alton's book, Unified Fitness. ${ }^{27}$ To learn the elements of the qigong, military participants began with private one-hour sessions with Alton four times a week for the first two weeks. The schedule for this two-week period is shown in Table 1. Once the full routine had been learned, participants attended 20-minute group sessions (two to three in a group) three times a week for six weeks. To support the instruction, participants were each given a DVD of Alton's RE qigong routine to guide them in practicing qigong on days when there were no scheduled sessions.

\section{Data Analysis}

Before data analysis, the principle investigator created a log bracketing all preconceived ideas regarding the experiences, effects, and outcomes that the qigong intervention may have on the study participants. The purpose of this step was to aid the investigator in separating the actual experiences of the partici- 
Table 1. Elements of Reflective Exercise Taught in the First 2 Weeks

\begin{tabular}{ll}
\hline Session & \multicolumn{1}{c}{ Element } \\
\hline Session 1 & Learn reverse breathing \\
Session 2 & $\begin{array}{c}\text { Practice reverse breathing and learn } \\
\text { independently the hand and leg aspects of } \\
\text { the six subtle movements } \\
\text { Practice reverse breathing and integrate six } \\
\text { subtle movement hand and leg aspects with } \\
\text { reverse breathing } \\
\text { Practice the six subtle movements with } \\
\text { reverse breathing } \\
\text { Practice the six subtle movements and learn } \\
\text { standing sensory meditation involving hand } \\
\text { movements and seated or reclining static } \\
\text { sensory meditation } \\
\text { Practice the six subtle movements, learn more } \\
\text { standing sensory meditation involving whole } \\
\text { body movement that focuses on the hip and } \\
\text { pelvic region, and practice seated or } \\
\text { reclining static sensory meditation } \\
\text { Practice the six subtle movements, expand the } \\
\text { whole-body standing sensory meditation to } \\
\text { include the chest and head regions, and } \\
\text { practice seated or reclining static sensory } \\
\text { meditation } \\
\text { Practice the full routine without interruption: } \\
\text { six subtle movements, whole-body standing } \\
\text { sensory meditation, and seated or reclining } \\
\text { static sensory meditation }\end{array}$ \\
& \\
&
\end{tabular}

pants from personal expectations and interpretation of the investigator. Data analysis then began following steps described by Giorgi $^{23}$ in applying Husserl's Descriptive Phenomenological methods. ${ }^{22}$ The first step of analysis involved reading all of the interview data to obtain a sense of the whole. During this step, the principal investigator read and reread the transcribed audio files in entirety to obtain an overall perspective on the data as a whole. The second step was to separate the data into meaning units $^{23}$ that represent key elements, terms, attitudes, and experiences. For this step, transcripts were examined individually and distinct expressions of specific thoughts or ideas were identified. During this step, NVivo 9 (QSR International, Burlington, MA) software was used to assist in identifying and organizing the key words and elements among participants' reported experiences. These meaning units were then cut from the original interviews and reorganized into similar meanings. The reorganization of the meaning units into groupings based on similarity resulted in the emergence of overarching themes. The third step was to transform the data into the language of the researcher to provide an analytic interpretation of the meaning of the units. The final step of the analysis was to synthesize the transformed meaning units into a consistent statement that described the experiences of service members with mTBI learning and practicing qigong. Although data saturation was not a goal in this pilot study before we began to analyze data, by the fifth participant, no new themes were identified, indicating that data saturation was likely achieved.

\section{RESULTS}

The study sample consisted of six married or engaged-to-be married, male military service members ranging in age from 21 to 48 years with mTBI acquired during active-duty service. Of the six participants who began the qigong intervention, five completed the full eight-week intervention. Stating time constraints as the reason, one participant withdrew from the study after four weeks of instruction. The participants varied by branch of military service: active Army (2), active Marines (1), reserve Army (1), reserve Marines (1), and Army National Guard (1). Four general themes emerged from the interview data: "the physical experiences of qigong," "regaining control," "no pain, a lot of gain," and "barriers to practice of qigong."

\section{Theme 1: The Physical Experiences of Qigong}

All participants reported feeling physical sensations during qigong practice. These were described in a variety of ways, including the sensation of static electricity running through the hands and arms, a pulse going up and down the body from head to groin, and a pressure in the head and sinuses occasionally resulting in a "pop" similar to that experienced when changing altitude. One participant offered this description: "We went through the whole exercise and while I was sitting there meditating and had the pulse coming up and down, it's almost like I felt a lightning bolt shoot through my chest." Another stated that "It feels like static electricity. Mostly it's strongest coming out of my head, but it's throughout my whole body." These strong physical sensations were surprising to the participants. One noted "A couple of weeks after [starting], I could feel [energy] going through my veins and stuff and that freaked me out. I didn't know what that was." Another participant described in more detail similar responses when practicing the qigong.

It almost feels like I have a big ball of energy in my hands ... both of my hands get to tingling ... it feels like I'm pushing a force field. I hold them [hands] together and pull them apart; it feels like it's pulling them towards each other. And the first time that happened that freaked me out ... That's your inner energy ... It amazes me.

In addition to experiencing physical energy sensations, participants used a variety of words to describe the overall experience of practicing qigong. They used words such as "peaceful," "relaxing," and "calming," and they likened the back and forth elements of the six subtle movements to being in a tide or on the ocean. The participants' comments and descriptions were compelling given that all six expressed a degree of skepticism about the possible effects that qigong may offer before starting the intervention. One participant explained, "Yeah, at first I was like, I don't know, it sounds kind of weird; I don't know if it will work." Despite their initial reservations regarding qigong, the participants remained sufficiently open-minded, allowing for a dramatic reversal of opinion. Specifically, one participant exclaimed, "I'm blown away by this [qigong]." 


\section{Theme 2: Regaining Control}

A dramatic element of the qigong experience was the sense of empowerment that the participants gained during the period of the intervention. During baseline interviews, many of the participants expressed feeling a loss of control with regard to their injury and recovery, a common phenomenon among those with comorbid PTSD. ${ }^{9}$ After learning and practicing qigong, dramatic changes were evident in how the participants viewed themselves. Most described an ability to control their mTBIrelated symptoms through the use of qigong. One participant described the use of qigong in managing his chronic headaches.

The pulse had gotten to my head where I actually hit [it] and had the brain injury. I've had it come up and it felt like a big knot of pressure and then once I [brought] that pulse up to my head it felt like it just all evenly flowed away and then I didn't have [any] pain in my head.

Another participant described a similar reaction when he was experiencing a migraine headache.

I had a real bad migraine when we started. I told him [the qigong master] about it and then, about halfway through the meditation of bringing that pulse up into my head and down, I felt the relief of pressure and ever since then my headaches [have] been gone.

Participants also discussed how they were able to use qigong to calm their feelings of anxiety. One participant noted "Any time I get stressed out, I can do the movements [qigong] and it's like my stress is gone."

Qigong also had a positive impact on sleep quality as revealed by one participant who shared the following:

I would wake up periodically in the middle of the night just because I was antsy. Sometimes I'd lay there for 1 or 2 hours. My brain would always be racing thoughts, and it was like I just couldn't go to sleep. And now I do this [qigong] routine once before I go to bed and . . . I got a clear mind. I'm just relaxed and it takes 5 minutes to fall asleep and I'll sleep throughout the whole night.

All except one of the participants in this study noted an ability to practice meditation without the significant flashbacks and intrusions of PTSD when they incorporated breathing and movement techniques from their qigong practice. One participant described the following regarding his flashbacks:

During the movement, I'm concentrating on my breath, and the movement, and just relaxing ... I haven't had any of the flashbacks during movement. I haven't had any visions during movement. It's a very relaxed, peaceful feeling.

Another participant described the process of gaining control over his flashbacks although the process occurred more gradually.

In the first couple of sessions of meditation, intruding thoughts would come in, but the way that it's actually in- troduced, with the movements and everything, it kind of sort of blocks them out. So, I say by the fourth or fifth session, I was able to sit down and meditate and not have thoughts whatsoever that were negative.

For the participant who was still experiencing flashbacks, he described a change in the experience to one that he could control and that was far less intense than previous flashbacks.

When I do the meditation, I almost expect a flashback to happen ... They have become fewer, I'll say that, and I don't know if that's because of the qigong, but I'm going to say it's part of it. It's OK for it [the flashback] to happen [during qigong]; this is my safe place.

Other comments by the participants support the idea of gaining control and experiencing a sense of empowerment, as reflected in the following comment: "It's like being a Kung Fu martial artist." Another participant noted "Some of the things are really amazing ... I never thought I could control [my body]. I never thought that was possible." Another participant exclaimed, "Just by going through this, I feel like I have control of myself again. I don't get angered as easy.” And another observed about himself "It's like I'm more happy, more aware, more controlled about myself than I ever was." Perhaps one of the most dramatic comments came from a participant who had gained the ability to control his headaches and discontinue his pain medication.

I'm used to people throwing drugs at you. Pills solve everything nowadays. That's how everybody sees it. When [qigong] was first introduced to me, I never heard of it. I was like, "There's no way you could take just your mind and your body and do a routine and exercise and learning to control yourself to heal something." I was like, "That's bull crap.” But now that I've gotten this deep into [qigong], for me to be able to take away a headache on my own by just controlling blood flow going to my brain ... that tells me right there I don't need an Excedrin or a Tylenol or some kind of drug to get rid of that.

\section{Theme 3: No Pain, a Lot of Gain}

Participants were asked whether they thought that the practice of qigong had affected their responses to the various rehabilitation treatments that they had been receiving concurrently with the qigong. All participants were in agreement that the practice of qigong had a positive effect on their course of recovery, albeit more for some than others. Some discussed that by practicing the qigong routine before their neurocognitive exercises, they were more relaxed and able to focus on the task at hand instead of being distracted as they had been before learning qigong. Three participants expressed their belief that qigong had accelerated their rehabilitation process and allowed for an earlier discharge than may have been possible for them without qigong practice. One participant reported the following about his perceptions regarding the qigong intervention.

It sped [my recovery] up a whole lot because before I started, I was still having a little bit of memory problems 
and everything... But after doing this, my rehab has sped up so quickly that they're actually going to be discharging me [a month ahead of schedule].

Another participant related comments that his neurorehabilitation therapists had shared with him describing changes that they had observed in his behaviors.

They've [the therapists] said I seem a whole lot happier and joyful in life than what I used to be. They said I'm less depressed. I'm less sad. I'm more likely to go out now than sit at home and rock. I always want to go places now. My overall happiness has doubled. So that's dramatically changed my life and my whole perspective.

During a period of eight weeks, the service members gained a new life skill with relative ease. Five of the six service members stated that they experienced no difficulty in learning the full qigong routine despite having limited capabilities related to balance, concentration, and/or memory-a notion they attributed to the simplicity and brevity of RE qigong. Most importantly, all expressed that having the qigong master available to direct and guide the class sessions as opposed to learning solely from a DVD was a big part of their acceptance. This allowed them to receive definitive answers to their questions, explanations for the rationale of elements of practice, improved technique through immediate feedback if they were doing elements incorrectly, and also to be held accountable for their practice.

All of the participants found short-term benefits and reported that they would recommend qigong to others. They stated that the level of discipline needed to practice RE qigong was well suited to military personnel and that qigong or a similar modality such as tai chi would be an ideal offering to service members with $\mathrm{mTBI}$, given the amount of downtime, in particular, for those assigned to Warrior Transition Units.

\section{Theme 4: Barriers to Practice of Qigong}

Participants did experience barriers related to their qigong practice. The most commonly discussed barriers were distractions during practice. Although effort was made to minimize this by having the participants practice in a building outside of the resident house, some distractions, particularly noises and interruptions, could not be eliminated entirely. After a few weeks of practice, some of the participants were able to block the distractions, as revealed by one participant who noted, "Today I meditated outside and you got birds and you got cars going by and everything. But I've learned actually to block them out and just concentrate on what I'm doing."

Some service members discussed a sense of self-consciousness during the practice, which was a bigger concern for some than others. One participant stated, "I don't think I'd be a fan of doing [qigong] in big groups. You'd probably get a lot of funny looks." For the majority, this concern was diminished once they completed the learning process and had developed proficiency in the complete qigong exercise. One service member noted:

[The qigong master] said "It's a nice day. We can do [qigong] out in the backyard. Do you care if people see you?” I was like, "No, I don't care what people think.” I mean, you're practicing a form of ancient Chinese martial art. Why should you be ashamed of it?

Physical symptoms were another barrier the participants discussed. The symptoms included headaches, fatigue, and musculoskeletal pain, which were either a result of their mTBI event or, in some cases, from other previous injuries. In the case of severe headaches and fatigue, some qigong sessions needed to be rescheduled. Usually, however, the service members elected to "push through" the sessions with the qigong master despite headaches or fatigue because, in their personal experiences, they were confident that the qigong would lessen or relieve the headache and restore a better sense of well-being after the session.

One participant did not completed the full eight-week intervention (withdrew after four weeks), stating that lack of time was a barrier to the practice of qigong. He explained, "It [qigong] is time-consuming .... and with so many things happening it's hard for me to really get into a rhythm. So I'd rather not do it if I can't do it all of the way." That only one participant cited time as an obstacle was unexpected, given that time constraints are typically one of the most common barriers in the implementation of interventions that require active participation. Participants in this study, however, likely had more time to commit because they were separated from family and jobs while participating in the neurorehabilitation program.

Despite these barriers, participants overwhelmingly enjoyed and often looked forward to their qigong sessions and did not perceive the sessions as a burden, as illustrated by the following statement:

Every time I see [the qigong master] I just start smiling because I'm like, "How is this possible?" I ask him all the time. ... He tries to explain it to me and it just goes right over the top of my head. But I mean for me to sit there and [experience the benefits], I have a totally different point of view of it now.

\section{DISCUSSION}

The study participants stated that they experienced many general health improvements during the course of the qigong intervention. One of the most notable improvements was with general mood. These findings are similar to those of Blake and Batson, ${ }^{28}$ who conducted an eight-week qigong intervention in a nonmilitary population of individuals with TBI. In this British pilot study, the investigators concluded that individuals who participated in eight weeks of qigong instruction reported improvements in their overall mood as well as self-esteem. Other improvements that the service members described were consistent with qigong literature in non-TBI, nonmilitary study populations with regards to improved sleep, ${ }^{29}$ reduced impact of chronic pain syndromes, ${ }^{30-32}$ and decreased stress and anxiety. ${ }^{12}$

The findings of this study are also similar to those of Grodin and colleagues ${ }^{9}$ and their work with victims of torture, particularly Tibetan monks, with subsequent PTSD. The ability to calm the body through meditation has been recognized in Eastern culture for thousands of years. The physiologic impact that meditation has on calming noradrenergic hyperstimulation has been 
well established in medical literature since the 1970s. In working with Tibetan monks with PTSD, Grodin and colleagues observed that meditation, a fixture in the life of a monk, would often cause distress and anxiety as the result of intrusions and flashbacks while attempting to meditate rather than having its usual calming effect. When the monks practiced qigong, however, the act of incorporating the whole body into the practice of meditation through breathing and movement led to significantly fewer flashbacks than with traditional meditation. ${ }^{9,33}$ The military service members of this pilot study who suffered from comorbid PTSD in addition to their mTBI symptoms echoed these previously reported findings. They stated that they often experienced painful and vivid flashbacks during meditation, a standard component of their mTBI neurorehabilitation. Once they started practicing qigong, they too observed that they could obtain the benefits of meditation without the intrusions of PTSD.

This feasibility study enrolled a small number of participants drawn from a convenience sample of military service members with mTBI undergoing treatment in a civilian-based, outpatient neurorehabilitation program. Because of its qualitative nature, the findings from this study cannot be generalized beyond this population of service members with mTBI. The findings may be influenced, in part, by the nature of the personalities of the service members who were willing to participate and by the increased free time available to them because of separation from friends and family while undergoing rehabilitation. In addition, it is not possible to fully differentiate improvements attributable to qigong versus those resulting from standard rehabilitation therapies given the absence of a comparison group. Nonetheless, the acceptance and perceived benefit that service members attributed to qigong in this study supports future research to understand better the effects of this intervention.

Participants in this study offered profound examples of how qigong enabled them to take control of their present symptoms and improve their general outlook on the future. Their perceptions were that qigong was conducive to the highly disciplined mindset of military service members and that the simplicity of the qigong form used in this study, Reflective Exercise, was well suited to those with symptoms associated with mTBI. For this reason and given the high safety profile of qigong, one practical implication of the research is the implementation of similar RE qigong programs in Warrior Transition Units where service members may have dedicated time to commit to initially learning qigong. Another implication of this study is the need for additional research into the phenomenon described by the participants of qigong practice rather than medications to address symptoms such as headache, insomnia and anxiety. If such effects can be demonstrated in experimental research, the impact may go beyond decreasing medication use and risk of polypharmacy. Qigong may have the added benefit of promoting empowerment and control in a population for whom elements of control have been lost following a brain injury.

\section{Acknowledgments}

The authors thank the Defense and Veterans Brain Injury Center (DVBIC) and its Charlottesville facility leadership: Dr Don Nidiffer, Dr Jeffrey Barth, and Dr Tina Trudel, as well as all of the providers and staff of the DVBIC-Charlottesville Rehabilitation Program who were integral in facilitating this research. This research was sponsored by the TriService Nursing Research Program grant \#HU0001-1-10-TS13, Uniformed Services University of the Health Sciences; however, the information or content and conclusions do not necessarily represent the official position or policy of, nor should any official endorsement be inferred by, the TriService Nursing Research Program, the Uniformed Services University of the Health Sciences, the Department of Defense, or the U.S. government.

\section{REFERENCES}

1. Terrio H, Brenner LA, Ivins BJ, et al. Traumatic brain injury screening: preliminary findings in a US army brigade combat team.J Head Trauma Rehabil. 2009;24:14-23.

2. Sayer NA, Chiros CE, Sigford B, et al. Characteristics and rehabilitation outcomes among patients with blast and other injuries sustained during the global war on terror. Arch Phys Med Rehabil. 2008; 89:163-170.

3. Galarneau MR, Woodruff SI, Dye JL, Mohrle CR, Wade AL. Traumatic brain injury during operation Iraqi freedom: findings from the united states Navy-marine corps combat trauma registry. J Neurosurg. 2008;108:950-957.

4. Management of Concussion/mTBI Working Group. VA/DoD Clinical Practice Guideline for Management of Concussion/Mild Traumatic Brain Injury (mTBI). In: Affairs DoV, Defense Do, eds. Washington, DC: The Office of Quality and Performance Quality Management Directorate; 2009:109.

5. Tanielian T, Jaycox LH, Schell TL, et al. Invisible Wounds of War: Psychological and Cognitive Injuries, Their Consequences and Services to Assist Recovery. Washington, DC: The Rand Centre Military Health Policy Research; 2008.

6. U.S. Department of Defense Task Force on Mental Health. An Achievable Vision: Report of the Department of Defense Task Force on Mental Health. Falls Church, VA: U.S. Department of Defense Task Force on Mental Health; 2007.

7. Dao J, Frosch D. Feeling warehoused in army trauma care units. The New York Times. April 24, 2010.

8. Huggins JM, Brown JN, Capehart B, Townsend ML, Legge J, Melnyk SD. Medication adherence in combat veterans with traumatic brain injury. Am J Health Syst Pharm. 2011;68:254-258.

9. Grodin MA, Piwowarczyk L, Fulker D, Bazazi AR, Saper RB. Treating survivors of torture and refugee trauma: A preliminary case series using qigong and T'ai chi. J Altern Complement Med. 2008;14:801806.

10. Cheung BM, Lo JL, Fong DY, et al. Randomised controlled trial of qigong in the treatment of mild essential hypertension. J Hum Hypertens. 2005;19:697-704.

11. Lee MS, Pittler MH, Guo R, Ernst E. Qigong for hypertension: a systematic review of randomized clinical trials. J Hypertens. 2007;25: 1525-1532.

12. Skoglund L, Jansson E. Qigong reduces stress in computer operators. Complement Ther Clin Pract. 2007;13:78-84.

13. Lee MS, Lee MS, Choi ES, Chung HT. Effects of qigong on blood pressure, blood pressure determinants and ventilatory function in middle-aged patients with essential hypertension. Am J Chin Med. 2003;31:489-497.

14. Newberg AB, Iversen J. The neural basis of the complex mental task of meditation: neurotransmitter and neurochemical considerations. Med Hypotheses. 2003;61:282-291. 
15. Ryu H, Jun CD, Lee BS, Choi BM, Kim HM, Chung HT. Effect of qigong training on proportions of $\mathrm{T}$ lymphocyte subsets in human peripheral blood. Am J Chin Med. 1995;23:27-36.

16. Yang Y, Verkuilen J, Rosengren KS, et al. Effects of a Taiji and qigong intervention on the antibody response to influenza vaccine in older adults. Am J Chin Med. 2007;35:597-607.

17. Xin L, Miller YD, Brown WJ. A qualitative review of the role of qigong in the management of diabetes. J Altern Complement Med. 2007;13:427-433.

18. Vera FM, Manzaneque JM, Maldonado EF, et al. Biochemical changes after a qigong program: lipids, serum enzymes, urea, and creatinine in healthy subjects. Med Sci Monit. 2007;13:CR560CR6.

19. Kennedy JE, Jaffee MS, Leskin GA, Stokes JW, Leal FO, Fitzpatrick PJ. Posttraumatic stress disorder and posttraumatic stress disorderlike symptoms and mild traumatic brain injury. J Rehabil Res Dev. 2007;44:895-919.

20. Brenner LA, Ivins BJ, Schwab K, et al. Traumatic brain injury, posttraumatic stress disorder, and postconcussive symptom reporting among troops returning from Iraq. J Head Trauma Rehabil. 2010;25: 307-312.

21. Schneiderman AI, Braver ER, Kang HK. Understanding sequelae of injury mechanisms and mild traumatic brain injury incurred during the conflicts in Iraq and Afghanistan: persistent postconcussive symptoms and posttraumatic stress disorder. Am J Epidemiol. 2008; 167:1446-1452.

22. Husserl E. Ideas: General Introduction to Pure Phenomenology. New York: Macmillan; 1962.
23. Giorgi A. Phenomenology and Psychological Research. Pittsburgh, PA: Duquesne University Press; 1985.

24. Morse JM. Determining sample size. Qual Health Res. 2000;10:3-5.

25. Creswell JW. Qualitative Inquiry and Research Design: Choosing among Five Traditions. Thousand Oaks, CA: Sage Publications; 1998.

26. Morse JM. Critical Issues in Qualitative Research Methods. Thousand Oaks, CA: Sage Publications; 1994.

27. Alton J. Unified Fitness: A 35 Day Exercise Program for Sustainable Health. Charlottesville, VA: Hampton Roads Publishing Company; 2002.

28. Blake H, Batson M. Exercise intervention in brain injury: a pilot randomized study of Tai chi Qigong. Clin Rehabil. 2009;23:589598.

29. Craske NJ, Turner W, Zammit-Maempe J, Lee MS. Qigong ameliorates symptoms of chronic fatigue: a pilot uncontrolled study. Evid Based Complement Alternat Med. 2009;6:265-270.

30. Lansinger B, Larsson E, Persson LC, Carlsson JY. Qigong and exercise therapy in patients with long-term neck pain: a prospective randomized trial. Spine. 2007;32:2415-2422.

31. Lee MS, Pittler MH, Ernst E. External qigong for pain conditions: a systematic review of randomized clinical trials. J Pain. 2007;8:827831.

32. Yang KH, Kim YH, Lee MS. Efficacy of QI-therapy (external Qigong) for elderly people with chronic pain. Int J Neurosci. 2005; 115:949-963.

33. Benedict AL, Mancini L, Grodin MA. Struggling to meditate: contextualising integrated treatment of traumatised Tibetan refugee monks. Ment Health Relig Cult. 2009;12:485-499. 